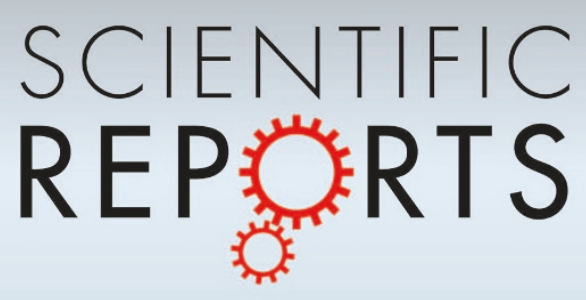

OPEN

SUBJECT AREAS:

FLUID DYNAMICS

MECHANICAL ENGINEERING

Received

9 January 2014

Accepted

24 March 2014

Published

24 April 2014

Correspondence and requests for materials should be addressed to R.G. (rama@tifrh.res.

in)

* Current address: TIFR Centre for Interdisciplinary

Sciences, Tata Institute of Fundamental

Research, Narsingi, Hyderabad 500075, India. On lien from the

Jawaharlal Nehru Centre for Advanced Scientific Research, Bangalore.

\section{Why a falling drop does not in general behave like a rising bubble}

\author{
Manoj Kumar Tripathi, Kirti Chandra Sahu \& Rama Govindarajan*
}

Department of Chemical Engineering, Indian Institute of Technology Hyderabad, Yeddumailaram 502 205, Andhra Pradesh, India.

Is a settling drop equivalent to a rising bubble? The answer is known to be in general a no, but we show that when the density of the drop is less than 1.2 times that of the surrounding fluid, an equivalent bubble can be designed for small inertia and large surface tension. Hadamard's exact solution is shown to be better for this than making the Boussinesq approximation. Scaling relationships and numerical simulations show a bubble-drop equivalence for moderate inertia and surface tension, so long as the density ratio of the drop to its surroundings is close to unity. When this ratio is far from unity, the drop and the bubble are very different. We show that this is due to the tendency for vorticity to be concentrated in the lighter fluid, i.e. within the bubble but outside the drop. As the Galilei and Bond numbers are increased, a bubble displays underdamped shape oscillations, whereas beyond critical values of these numbers, over-damped behavior resulting in break-up takes place. The different circulation patterns result in thin and cup-like drops but bubbles thick at their base. These shapes are then prone to break-up at the sides and centre, respectively.

sk a fluid mechanician the following question: If a bubble of density $\rho_{1}$ rises in a fluid of density $\rho_{2}$, would a drop of density $\rho_{2}$ rise in fluid of density $\rho_{1}$ in the same way, if gravity were reversed? The answer is likely to be a yes (except from experts on the topic), with a caveat that viscosity must be appropriately reassigned. The correct answer is $\mathrm{a} \mathrm{no}^{1}$, and in this paper we study the physics behind this difference. We show how vorticity tends to be concentrated in the lighter fluid, and this changes the shape and the entire behavior. By the term bubble we mean a blob of fluid which is lighter than its surroundings, and by drop a fluid blob that is heavier than its surroundings. We discuss the limit in which a drop and bubble can behave the same, and present two alternatives in this limit to design an equivalent drop for a bubble.

Due to their importance in industry and in natural phenomena, and due to their appeal to our scientific curiosity, bubbles and drops have been studied for a long time. The literature on this subject is therefore huge, with several review articles by several authors ${ }^{2-4}$ and others. Appealing introductions to the complexity associated with bubble and drop phenomena can also be found in Refs. 5, 6. There are yet many unsolved problems, which are the subject of recent research (see e.g. ${ }^{7-13}$ ). Bubbles and drops have often been studied separately, e.g. ${ }^{11,14-18}$ on bubbles and ${ }^{19-24}$ on drops. However, there is also a considerable amount of literature which discusses both together, e.g. ${ }^{25-30}$.

When the bubble or drop is tiny, it merely assumes a spherical shape, attains a terminal velocity, and moves up or down. An empirical formula for the terminal velocity of small air bubbles was found by Allen ${ }^{31}$ in 1900 . Later, two independent studies by Hadamard ${ }^{25}$ and Rybczynski ${ }^{32}$ led to the first solution for the terminal velocity and pressure inside and outside of a slowly moving fluid sphere in another fluid of different density and viscosity. The spherical vortex solution due to $\mathrm{Hill}^{33}$ has been a keystone for most of the analytical studies on the subject. Later studies ${ }^{1}$ showed that at low Reynolds and Weber numbers, drops and bubbles of same size behave practically the same way as each other, both displaying an oblate ellipsoidal shape.

Bigger drops and bubbles are different. A comparison of bubble and drop literature will reveal that in the typical scenario, bubbles dimple in the centre ${ }^{14,34}$ while drops more often attain a cup-like shape ${ }^{13,35}$. This difference means that drops and bubbles which break up would do so differently. The dimples of breaking bubbles run deep and pinch off at the centre to create a doughnut shaped bubble, which will then further break-up, while drops will more often pinch at their extremities. A general tendency of a drop is to flatten into a thin film which is unlike a bubble. There could be several other modes of breakup $\left(\operatorname{see}^{35}\right)$ like shear and bag breakup for drops falling under gravity and catastrophic breakup at high speeds.

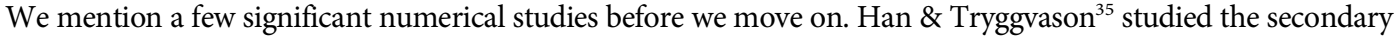
break-up of drops for low density ratios, whereas Tomar et al. ${ }^{56}$ investigated the atomization involving the break-up of liquid jet into droplets. We also note that recent developments in the field of lattice Boltzmann simulations ${ }^{54,55}$ allow one to simulate multiphase flows with high density ratio by using a clever doubly-attractive pseudo-potential. 


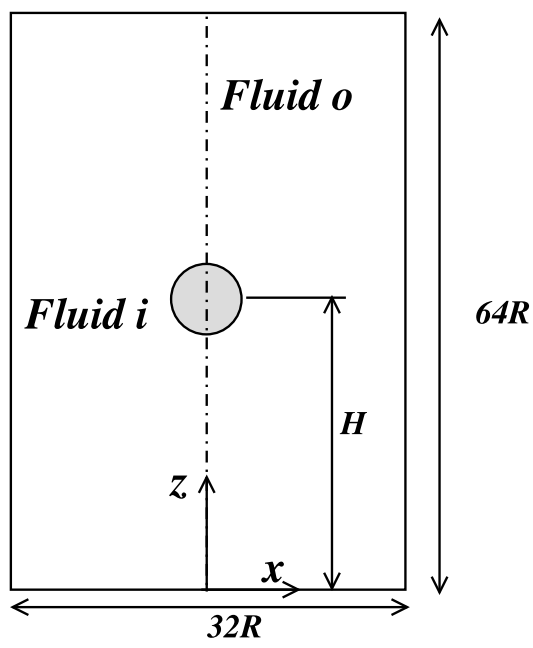

Figure $1 \mid$ Schematic diagram showing the flow domain as a box surrounding a bubble/drop and the axis of symmetry as a dot-dashed line. The outer and inner fluid phases are designated as ' $o$ ' and ' $i$ ', respectively.

We now pose the problem and discuss our approach to its solution.

Problem formulation and approach. The geometry and equations. The drop or bubble is initially spherical with radius $R$ and has viscosity and density $\mu_{i}$ and $\rho_{i}$ respectively, which are different from the respective quantities $\mu_{o}$ and $\rho_{o}$ for the surrounding fluid. The fluids are assumed to be immiscible, incompressible and Newtonian. Figure 1 is a schematic diagram of the initial configuration, where $x$ and $z$ represent the horizontal (radial) and vertical (axial) directions, respectively. The subscripts ' $i$ ' and ' $o$ ' stand for inner and outer fluid, respectively, $\sigma$ is the surface tension coefficient between the two fluids and $g$ the acceleration due to gravity. We study an axisymmetric flow, with the velocity and pressure fields denoted by $\mathbf{u}(u, w)$ and $p$ respectively, wherein $u$ and $w$ are the velocity components in the $x$ and $z$ directions, respectively. We treat the flow as isothermal.

Given the physical quantities in the problem, the Buckingham-pi theorem requires four independent dimensionless parameters to completely describe the system and the ones we choose are the density ratio $r$, the viscosity ratio $m$, the Galilei number $G a$ and the Bond

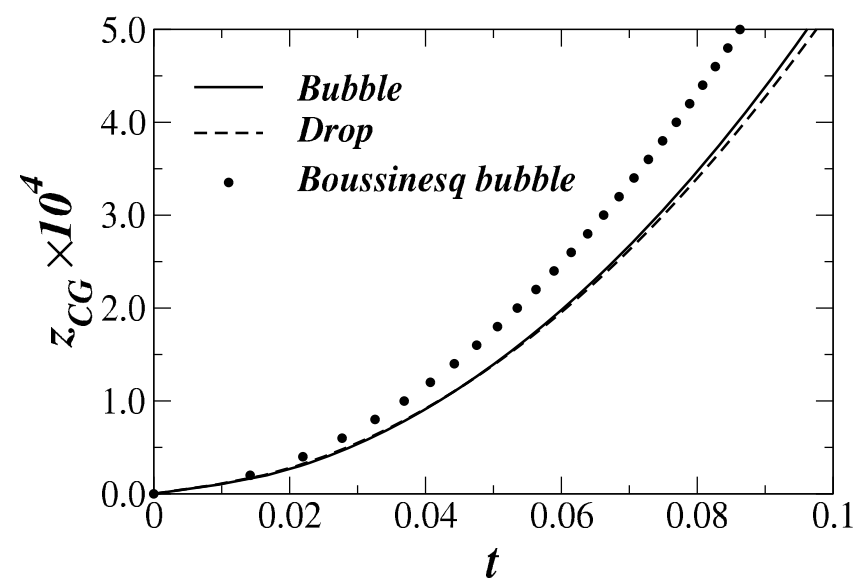

Figure $2 \mid$ Vertical location of the center of gravity as a function of time for a drop $(r=1.214$ and $m=76)$, an equivalent bubble based on Boussinesq approximation $(r=0.786$ and $m=76)$ and an equivalent bubble based on conditions (17) and (18) $(r=0.85$ and $m=0.1)$. The rest of the parameters are $G a=6$ and $B o=0.0005$.

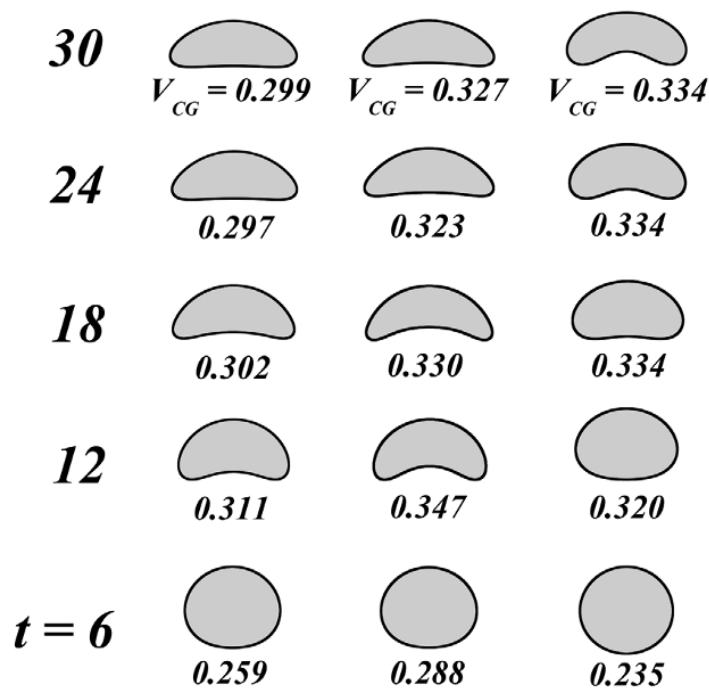

Figure $3 \mid$ Evolution of (a) bubble shape with time for $r=0.9, m / r=0.56$. (b) drop shape with time for $r=1.125, m / r=0.56$. (c) drop shape with time for $r=1.125$, with viscosity obtained from equation (17). The direction of gravity has been inverted for the drop in order to compare the respective shapes with those of the bubble. In all three simulations, $G a=$ $50, B o=50$, and the initial shape was spherical.

number $B o$. These are defined respectively as $r \equiv \rho_{i} / \rho_{o}, m \equiv \mu_{i} / \mu_{o}$, Ga $\equiv \rho_{o} g^{1 / 2} R^{3 / 2} / \mu_{o}$ and $B o \equiv \rho_{o} g R^{2} / \sigma$. The governing equations are thus non-dimensionalised using $R, \sqrt{g R}, \mu_{o}$ and $\rho_{o}$ as length, velocity, viscosity and density scales, respectively. Note that the Galilei number is like a Reynolds number, but defined by the gravity velocity scale rather than the actual forward velocity of the bubble. Since the latter is unknown a priori, and depends on several physical quantities, the present velocity scale is a cleaner one to adopt, and we do so in our study. For our discussions however it will be useful also to define a Reynolds number $R e \equiv \rho_{o} U_{0} R / \mu_{o}$ and a Weber number and $\mathrm{W} e \equiv \rho_{o} U_{0}^{2} R / \sigma$, where $U_{0}$ is a typical bubble velocity. Note that each of the four dimensionless parameters, when independently varied can change the dynamics of the blob. Along with the continuity equation:

$$
\nabla \cdot \mathbf{u}=0
$$

the momentum equation can be written with a continuum model for surface tension forcing $\left(\sec ^{36}\right)$ as

$\frac{D \mathbf{u}}{D t}=-\frac{\nabla p}{\rho}+\frac{1}{\rho \mathrm{Ga}} \nabla \cdot\left(\mu\left(\nabla \mathbf{u}+\nabla \mathbf{u}^{T}\right)\right)-\hat{\mathbf{j}}+\frac{\nabla \cdot \hat{\mathbf{n}}}{\rho \mathrm{Bo}} \delta\left(\mathbf{x}-\mathbf{x}_{\mathrm{s}}\right) \hat{\mathbf{n}}$,

wherein $\hat{j}$ is the unit vector in the vertically upward direction; $\delta$ is the Dirac-delta function, $\vec{x}_{s}$ a location on the interface and $\mathbf{n}$ its unit normal and $D[\equiv \partial / \partial t+\vec{u} \cdot \nabla]$ is the material derivative. On the right hand side, we have the pressure, viscous, gravitational and surface tension forces per unit volume respectively. Note that $\rho$ and $\mu$ are now dimensionless quantities, respectively scaled by the density and viscosity of the outer fluid.

Thus the surrounding or outer fluid obeys

$$
\frac{D \mathbf{u}_{\mathbf{o}}}{D t}=-\nabla p_{o}+\frac{1}{\mathrm{Ga}} \nabla^{2} \mathbf{u}_{\mathbf{o}}-\hat{\mathbf{j}},
$$

whereas the inner fluid, namely the drop or bubble, satisfies

$$
\frac{D \mathbf{u}_{\mathbf{i}}}{D t}=-\frac{\nabla p_{i}}{r}+\frac{m}{r \mathrm{Ga}} \nabla^{2} \mathbf{u}_{\mathbf{i}}-\hat{\mathbf{j}},
$$

with the interfacial conditions at $\mathbf{x}=\mathbf{x}_{\mathbf{s}}$ being continuity in velocity and the relevant stress balance. The surface tension term appears in 


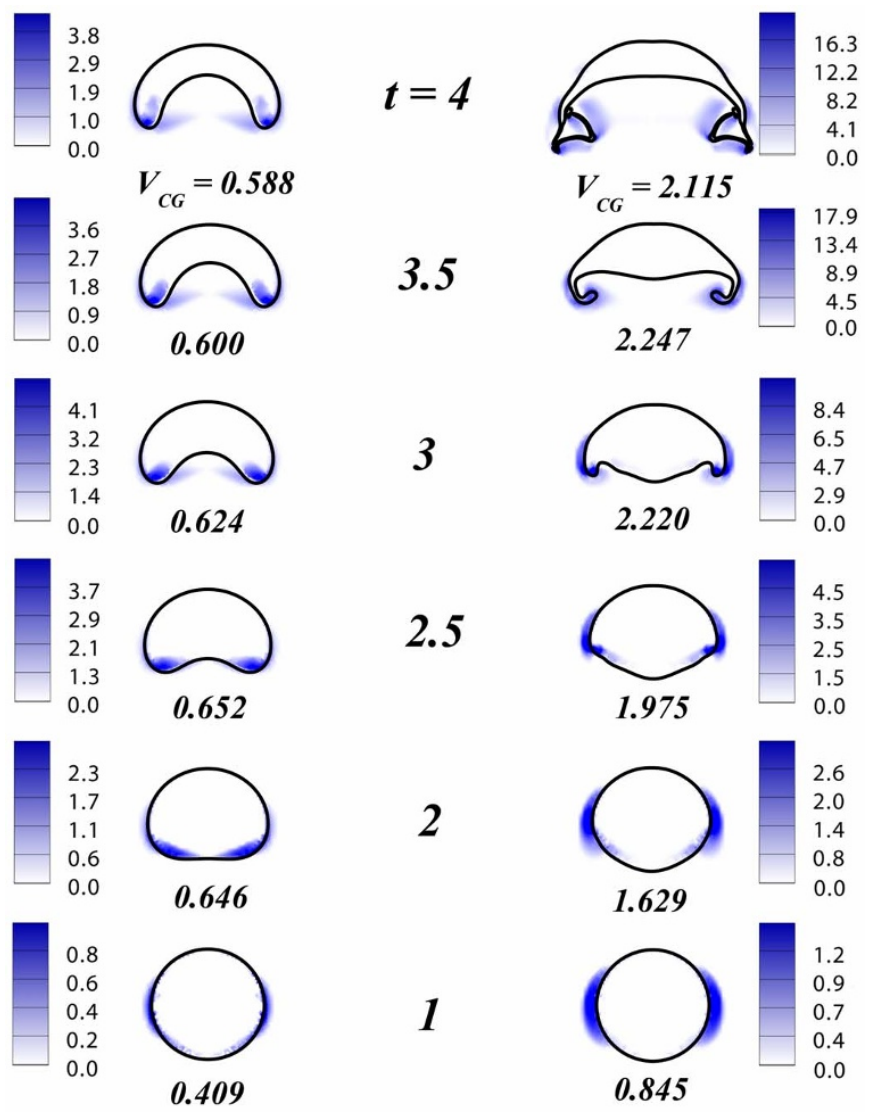

Figure 4 Evolution of (a) bubble and (b) drop shapes with time, when densities of outer and inner fluid are significantly different. As before, for the drop (right column), the direction of gravity has been inverted. In both simulations $G a=50$ and $B o=10$. The other parameters for the bubble system are $r=0.5263$ and $m=0.01$, while for the drop system $r=10$ and $m=0.19$. Note the shear breakup of the drop at a later time. Shown in color is the residual vorticity (Kolar, 2004).

the normal stress balance, which is $\left.p_{i}\right|_{s}=\left.p_{o}\right|_{s}+k / B o$ wherein $k$ is the local surface curvature defined as $k=\nabla \cdot \hat{\mathbf{n}}$.

Numerical simulations. In the framework of the diffuse-interface method $^{37}$, we express the surface tension term in the momentum equation (2) in the form

$$
\frac{\phi \nabla c}{\rho \mathrm{Bo}}
$$

and solve it along with the Cahn-Hilliard equation

$$
\frac{\partial c}{\partial t}+\mathbf{u} \cdot \nabla c=\frac{1}{\mathrm{Pe}} \nabla \cdot(M \nabla \phi)
$$

and the continuity equation (1). Here $c$ is the volume fraction of the outer fluid, $M=c(1-c)$ is the mobility, $\phi \equiv \epsilon^{-1} \sigma \alpha \Psi^{\prime}(c)-\epsilon \sigma \alpha \nabla^{2} c$ is the chemical potential wherein $\epsilon$ is a measure of interface thickness, $\Psi(c)=\frac{1}{4} c^{2}(1-c)^{2}$ is the bulk energy density and $\alpha$ is a constant; Pe $\equiv R V /\left(M_{c} \phi_{c}\right)$ represents the Péclet number wherein $M_{c}$ and $\phi_{c}$ are fixed characteristic values of mobility and chemical potential, respectively (see ${ }^{37}$ and references therein for more details). The dimensionless density and viscosity of the fluids are taken to be linear in $c$, as

$$
\rho=c+r(1-c), \quad \mu=c+m(1-c),
$$

where the value of $c$ is one and zero for outer and inner fluids, respectively.

Symmetry boundary conditions are applied at $x=0$ and Neumann boundary conditions are used at the other boundaries. To avoid numerical effects from the outer boundaries on the flow a computational domain of width $16 R$ and height $64 R$ is found to be sufficient for most of the cases, consistent with the study of Uno \& Kintner ${ }^{38}$. Where needed, larger domains have been used, and it has been checked that the results are independent of the domain size. In addition, the open source code Gerris, created by Stéphaen Popinet ${ }^{39}$ has been used for simulating drops which become very thin as compared to their initial radius. The adaptive grid refinement feature in Gerris was exploited for our benefit which saved a lot of time in our numerical study. The numerical method used and its validation are discussed in detail in the supplementary material. This code has previously been used extensively to study various miscible and immiscible flows (see e.g. Mishra et $\mathrm{al}^{40}$ ). The supplementary material contains more information about the numerical methods and comparisons with analytical results in the creeping and potential flow limits.

Drops versus bubbles: when must we discriminate? Let us first consider a solid spherical object falling through the fluid. Its equation of motion in Stokes flow (small $G a$ ), rendered dimensionless with the same scales as before, is

$$
G a \ddot{H}=\left[1-\frac{1}{r}\right] G a-\frac{9}{2 r} \dot{H}(1+O(G a)),
$$

where $H$ is the vertical location of the centre of the sphere, and a dot represents a time derivative. Maintaining $G a$ the same, it is easy to see that with gravity inverted and setting

$$
r_{b}=2-r_{d}
$$

a light solid bubble would attain the same steady state velocity. The subscripts $d$ and $b$ have been added to distinguish drop from bubble. Equation (8) makes it obvious however that in the transient phase, the accelerations of the bubble and drop must be different, which means that at steady state, the vertical locations of the drop and bubble will have a constant displacement. Note also that for every $r_{b}<1$, we can find a realistic $r_{d}$. However for $r_{d}>2$, there is no equivalent positive $r_{b}$. In other words, every solid bubble has an equivalent solid drop, but drops heavier than $r_{d}=2$ do not have an equivalent bubble.

The next question is, does the same relationship carry across to fluid bubbles and drops. The fact that the gradients in the viscous
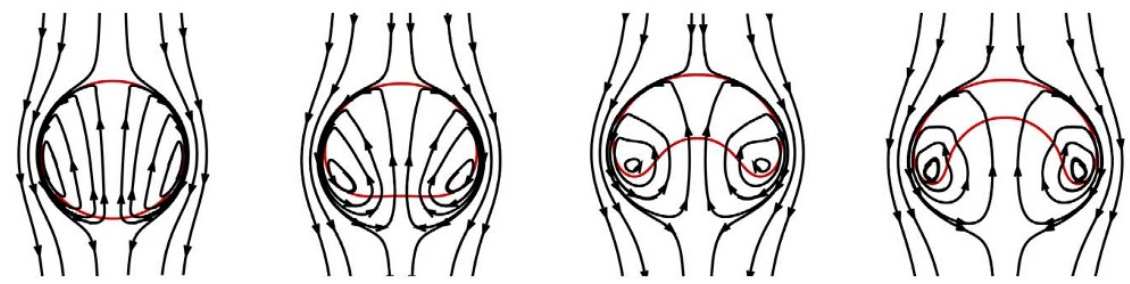

Figure $5 \mid$ Streamlines in the vicinity of a bubble for $t=1,2,3$ and 4 for parameter values $G a=50, B o=10, r=0.5263$ and $m=0.01$. The shape of the bubble is plotted in red. 

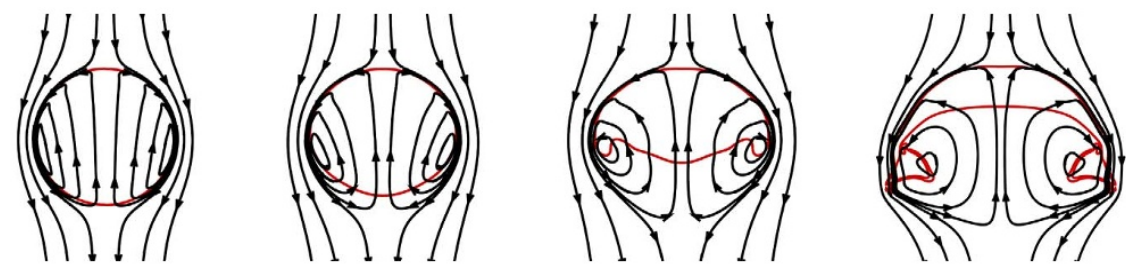

Figure 6 Streamlines in the vicinity of a drop for $t=1,2,3$ and 4 for parameter values $G a=50, B o=10, r=10$ and $m=0.19$. The shape of the bubble is plotted in red.

stresses and pressure inside the drop do not disappear any more makes the problem more complicated but, guided by Taylor and Acrivos $^{1}$ we may look for an equivalence in the limit $R e \ll 1$ and $W e \ll 1$, i.e., when we will have small inertia and a near-spherical drop. Hadamard's formula $\mathrm{a}^{25}$ for drag will apply in this case, and the equation of motion now is

$$
G a \ddot{H}=\left[1-\frac{1}{r}\right] G a-\frac{q(m)}{r} \dot{H}(1+O(G a)) .
$$

For a small or slowly moving drop, the $O(G a)$ terms can be neglected. For a Hadamard flow

$$
q(m)=\frac{3(2+3 m)}{2(1+m)},
$$

and it is seen that this expression reduces to the factor $9 / 2$ in equation (8) in the limit of infinite inner-fluid viscosity. Equation (10) is a first-order ordinary differential equation in $\dot{H}$, which can be integrated analytically. Given that the drop starts from rest, its velocity is

$$
\dot{H}_{d}=\frac{r_{d} G a_{d}}{q_{d}(m)}\left(1-\frac{1}{r_{d}}\right)\left[1-e^{\frac{-q_{d}(m)}{r_{d} G a_{d}} t}\right],
$$

and equivalently for a bubble with gravity reversed,

$$
\dot{H}_{b}=\frac{r_{b} G a_{b}}{q_{b}(m)}\left(\frac{1}{r_{b}}-1\right)\left[1-e^{\frac{-q_{b}(m)}{r_{b} G a_{b}} t}\right] .
$$

For the terminal velocities of the two to be the same, we must have

$$
\frac{r_{d} G a_{d}}{q_{d}}\left(1-\frac{1}{r_{d}}\right)=\frac{r_{b} G a_{b}}{q_{b}}\left(\frac{1}{r_{b}}-1\right) .
$$

For the outer fluid to have the same dynamics in drop and bubble cases, we must have, from equation (3),

$$
G a_{d}=G a_{b},
$$

while for the pressure jump across the interface to be the same in the two cases we need

$$
B o_{d}=B o_{b} .
$$

In addition, we would like the two to have the same history, i.e., to have been at the same position at the same time. This requirement is met for a Hadamard flow by setting

$$
\frac{r_{d}\left(1+m_{d}\right)}{2+3 m_{d}}=\frac{r_{b}\left(1+m_{b}\right)}{2+3 m_{b}},
$$

where we have made use of the expression (11). Using equation (17), equation (14) yields

$$
r_{d}=\frac{r_{b}}{2 r_{b}-1} .
$$

Keeping the Galilei numbers the same, condition (17) will yield positive values of $m_{d}$ for a given $m_{b}$ only when $r_{d}$ is less than a certain quantity. For the range $0.01<m_{b}<100$, we find $r_{d}$ must be less than $\sim 1.2$, and therefore $r_{b}>\sim 0.86$. Thus the equivalence applies only in a small range of density ratios. In other words, at low $G a$ and $B o$, a drop falling through a surrounding fluid of slightly lower density will display dynamics closely matching that of a corresponding bubble rising through slightly denser fluid. The dimensionless parameters of the drop and bubble are related by equations (15) to (18). On the other hand, as seen from equation (18), when $r$ is significantly different from 1 the dynamics of a drop should be qualitatively different from any bubble.

We now examine whether any equivalence is possible at higher $\mathrm{Ga}$ and $B o$ between a drop and bubble. The force balance, written in order of magnitude terms (in dimensional form) reads as

$$
\frac{\Delta p}{R} \sim O\left[\tilde{\rho} \frac{V^{2}}{R}\right]+O\left[\frac{\tilde{\mu} V}{R^{2}}\right]+O(g \Delta \tilde{\rho})+O\left[\frac{\sigma}{R^{2}}\right],
$$

where tildes in the superscript represent dimensional quantities. It is clear that for the pressure field in the surrounding fluid in the bubble and drop case to be the same, the nonlinear term in the velocity must be small, i.e., the flow must be gravity and not inertia dominated, or the Froude number, $V^{2} /(g R) \ll 1$. In this case, the drag scales as the forward velocity of the drop, so we have $V \sim \Delta \rho g R^{2} / \mu$, and we have
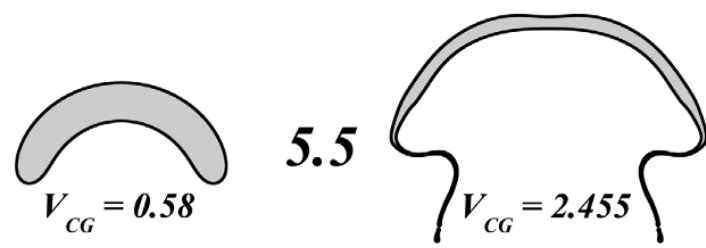

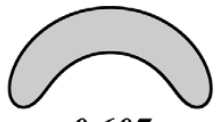

0.607

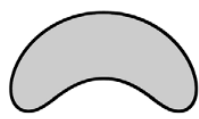

0.656
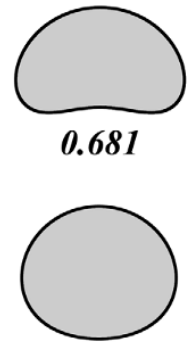

0.599
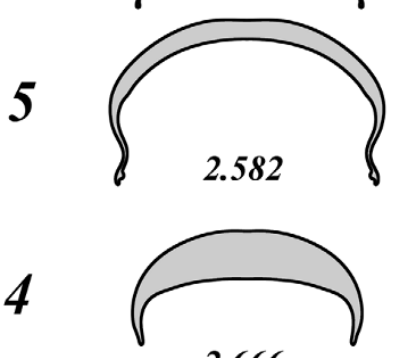

2.666
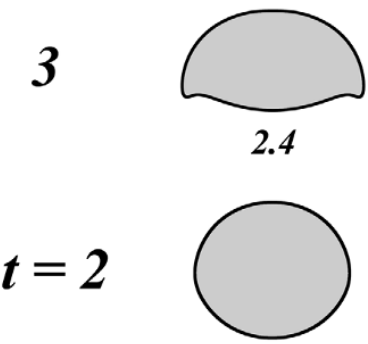

1.703
Figure $7 \mid$ Evolution of (a) bubble and (b) drop (gravity reversed) shapes with time. Parameters for both bubble and drop systems are: $G a=100$, $B o=50$ and $m=10$. The density ratio for the bubble and drop are $r=0.52$ and $r=13$ respectively, based on equation (18). 


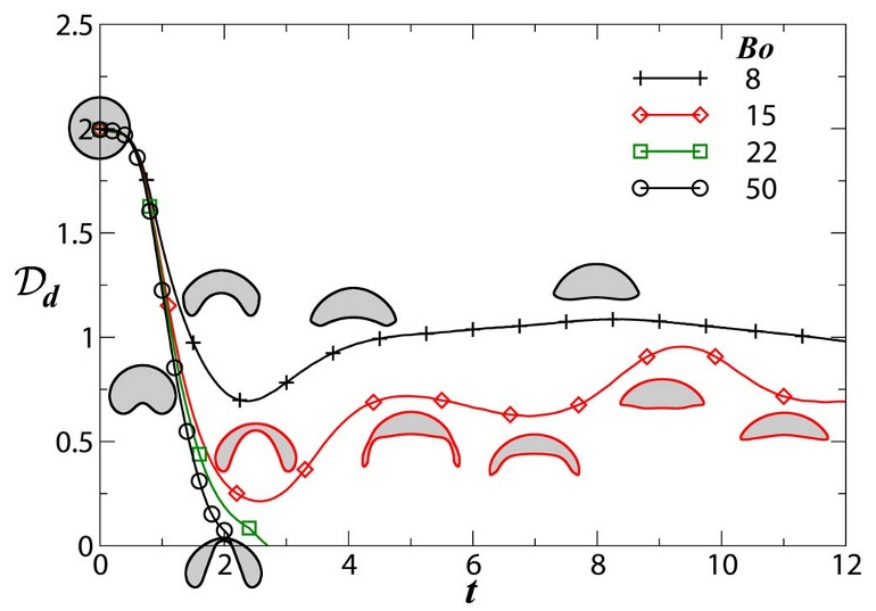

Figure 8 Variation of dimple distance versus time for $G a=50, r=$ $7.4734 \times 10^{-4}, \mathrm{~m}=8.5136 \times 10^{-6}$.

$$
(r-1)^{2} G a^{2} \ll 1
$$

Similarly, for surface tension to overwhelm inertial effects as far as the dynamics is concerned, and thus not allow for significant shape change of the drop, we must have $\rho V^{2} / R \ll \sigma / R^{2}$, yielding

$$
(r-1)^{2} G a^{2} \ll \frac{1}{r B o} .
$$

Thus an equivalence between a drop and bubble must be possible when $r \rightarrow 1$, even if $G a$ and $B o$ are not small, so long as inequalities (20) and (21) are satisfied. In this limit, it is usual to apply the Boussinesq approximation, by which the fact that the densities of the drop and the surrounding fluid are different will only affect the gravitational force term in the Navier-Stokes equation. In other words, $r$ is set to 1 everywhere except in the gravity term. For drops it was noted by Han \& Tryggvason ${ }^{35}$ that the Boussinesq approximation gives reasonable results for $1<r<1$.6. Interestingly, if we had begun by making the Boussinesq approximation, the NavierStokes equation would yield an equivalent bubble for a given drop with $r_{b}=2-r_{d}$ and $m_{b}=m_{d}$. We examine in the following section which of these choices will work better.

\section{Results}

Drop and bubble similarities and differences. In all cases where we compare drops and bubbles, the Galilei number and Bond number are kept the same for the bubble and the drop. In the case where inertia is small and surface tension is large, we saw that an equivalent bubble for every drop may be found when $r$ is close to 1 . We show in figure 2 the vertical location of the centre of gravity, nondimensionalised with the initial radius of the drop, of small inertia and high surface tension, with a density ratio $r=1.214$, and viscosity ratio $m=76.0$ as compared to its surroundings, obtained from numerical simulations. Also shown are the vertical locations of an equivalent bubble obtained from equations (17) and (18), and also one with $r_{b}=2-r_{d}$, and $m_{b}=m_{d}$. For ease of comparison, the direction of gravity is reversed for the drop in all the results presented. It is seen that the bubble obtained by equating the Hadamard drag produces a better equivalent bubble than one obtained by a priori making the Boussinesq approximation. All the blobs remain practically spherical till the end of the simulation shown.

At small inertia and moderate surface tension (not shown), bubbles and drops do not remain spherical, but with the density ratios close to 1 , and density and viscosity ratios related by equations (18) and (17), a bubble and drop display the same shape and dynamics as a function of time. We now consider bubbles and drops of higher inertia, where the inequalities (20) and (21) are not obeyed. Drop shapes obtained numerically for a density ratio close to unity are shown for this case in figure 3(b). The Galilei and Bond numbers are the same for the bubble and the drop, and the densities are related by equation (18). The Reynolds number based on the terminal velocity of the bubble and the drop is about 16 . The kinematic viscosity ratio $m / r$ for the bubble is kept the same as the drop in figure 3(a) and related by equation (17) in figure 3(c). It is apparent that a drop and its equivalent bubble behave qualitatively the same. The velocities of the drop and bubble are closer to each other when the viscosity relation (17) is used whereas the shapes are closer together when they have the same kinematic viscosity ratio.

When the density ratio is far from unity, no equivalence is possible. Equation (17) is no longer valid, nor possible to satisfy. We therefore compare drops and bubbles of the same $\mathrm{m} / \mathrm{r}$. Figure 4 makes it evident that neither the shape nor the velocity of the drop and bubble are similar to each other.

Shown in color in this figure is the residual vorticity $\Omega^{41}$, which is a good measure of the rotation in a flow. It is just the vorticity from which the shear part has been subtracted in a manner slightly different from that in the Okubo-Weiss parameter, and is discussed in greater detail in the supplementary material. Especially at later times in this figure, it is evident that residual vorticity is concentrated within the bubble but outside the drop. This is the primary difference between a bubble and a drop. The region of low pressure and high vorticity tends to lie in the lighter fluid. In the case of the bubble, this causes an azimuthally oriented circulation in the lower reaches, which then leads to a fatter base and aids in a pinch-off at the top of the bubble. In the case of a drop, the vorticity being outside means that the lower portion of the drop is stretched into a thin cylindrical sheet, and an overall bag-like structure is more likely. Also a pinch off in this sheet region is indicated rather than a central pinch-off. We present in figures 5 and 6 streamlines at various stages of evolution in this simulation. Closed streamlines are visible in the region of lower density, indicative of regions of maximum vorticity being located in the lighter fluid.

The fact that regions of low pressure and high vorticity would concentrate in the less dense fluid follows directly from stability arguments. A region of vorticity involves a centrifugal force oriented radially outwards, i.e., pressure increases as one moves radially outwards from a vortex. There is a direct analogy (see e.g. ${ }^{42}$ ) between
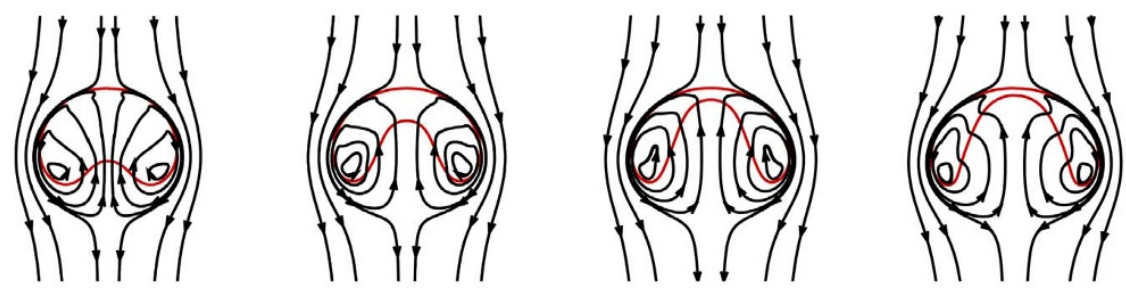

Figure $9 \mid$ Streamlines in and around the bubble at time, $t=1,1.5,2.0$ and 2.5 respectively, for $\mathrm{Ga}=50, \mathrm{Bo}=29, r=7.4734 \times 10^{-4}$ and $\mathrm{m}=8.5136 \times$ $10^{-6}$. The shape of the bubble is plotted in red. 

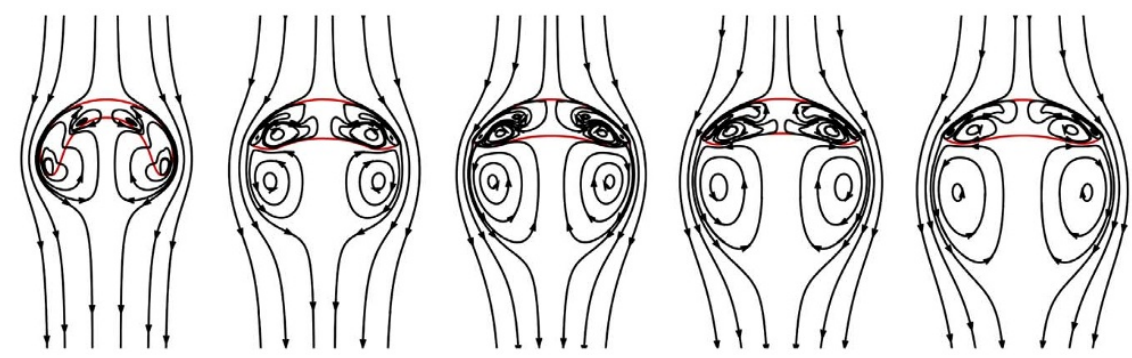

Figure $10 \mid$ Streamlines in and around the bubble at time, $t=2.5,5,7,9$ and 11 respectively, for $G a=50, B o=15, r=7.4734 \times 10^{-4}$ and $m=8.5136 \times$ $10^{-6}$.

density stratification in the vicinity of a vortex and in a standard Rayleigh Bénard flow. In the latter, we have a stable stratification when density increases downwards. In the former, we have a stable stratification when density increases radially outwards, i.e., when the vortex is located in the less dense region.

Figure 7 is a demonstration that for the same outer fluid even if the viscosity of the bubble and the drop were kept the same, and only the densities of the two were different, the behavior discussed above is still displayed.

Our results thus indicate that density is the dominant factor rather than viscosity in determining the shapes of inertial drops and bubbles. In particular, the vorticity maximum tends to migrate to the region of lower density, and this has a determining role in the shape of the structure. Since large density differences bring about this difference, these are effectively non-Boussinesq effects.

We note that given the large number of parameters in the problem, including initial conditions, which we have kept fixed, the location of maximum vorticity in the less dense region may not be universally observed in all bubble and drop dynamics. For example, the Widnall instability $^{43}$ in drops resembles the central break-up we have discussed. In the usual set-up of the Widnall instability, the densities of the inner and outer fluid are close to each other, so we may expect the drop to behave similar to a bubble, and the initial conditions are not stationary.

Our arguments above on vorticity migrating to the lighter fluid do not depend on gravity being present. We also confirm this in simulations which obtain the motion of a bubble and a drop started with a particular initial velocity in a zero-gravity environment. These are shown in the supplementary material.

Before breakup. Break-up of drops and bubbles is typically a threedimensional phenomenon on which much has been said, see e.g. the experimental studies of ${ }^{44-46}$ theoretical work of ${ }^{47,48}$ and numerical study of ${ }^{13}$. The transient behavior of liquid drops has been discussed extensively ${ }^{49-52}$, especially in the context of internal combustion engines, emulsification, froth-formation and rain drops. However, the transient behavior of bubbles has not been commented upon as much, and we make a few observations, regarding large-scale oscillations, that are not available in the literature to our knowledge. We note that since our simulations are restricted to axisymmetric break-up they may not always capture the correct break-up location or shape.

Various parameter ranges are covered in numerous papers in the past 100 years, and it is known that drops and bubbles break up at higher Bond numbers. The Bond number below which a bubble of very low density and viscosity ratio does not break, but forms a stable spherical cap, is about 8 (see e.g. ${ }^{53}$ ) which is similar to that found in our simulations. We begin by associating a time scale ratio with the Bond number. It may be said that surface tension would act to keep the blob together whereas gravity, imparting an inertia to the blob, would act to set it asunder. The respective time-scales over which each would act may be written as $T_{s}=\sqrt{\rho R^{3} / \sigma}$ for surface tension and $T_{g}=\sqrt{R / g}$ for gravity. The ratio

$$
\frac{T_{g}}{T_{s}}=\frac{1}{\sqrt{B o}}
$$

is a measure of the relative dominance. At $B o \gg 1$, surface tension is ineffective in preventing break-up, and we may expect a break-up at a time of $O(1)$, since we use gravitational scales. For $B o \sim 1$, it is reasonable to imagine a tug-of-war to be played out between surface tension and inertia in terms of shape oscillations, with a frequency of $O(1)$. Since a bubble usually breaks up at the centre by creating a dimple, the vertical distance $D_{d}$ of the top of the dimple from the top of the bubble is a useful measure to observe oscillations. Figure 8 shows the dimple distance as a function of time for various Bond numbers, and our expectations are borne out.

Figures 9 and 10 are typical streamline patterns in the vicinity of bubbles in the break-up and recovery cases respectively. Instantaneous streamlines are plotted by taking the velocity of the foremost point of the bubble as the reference, but the picture is qualitatively unchanged when the velocity of the centre of gravity of the drop is chosen instead. Both cases are characterized by a large azimuthal vortex developing within the bubble initially. In the break-up case (figure 9), this vortex is sufficient to cause the bubble surface to rupture and obtain a topological change, from a spherical-like bubble into a toroidal one. In all the cases of bubble recovery we have simulated, of which a typical one is shown (figure 10), there develop at later times several overlaying regions of closed streamlines, which act to counter the effect of rupture by the primary vortex, and to bring back the drop to a shape that is thicker at the centre. During each oscillation, we see the upper and lower vortices form and disappear cyclically.

A typical breaking drop, with its associated streamlines is shown in figure 11. As discussed above, the breakup is very different from that of a bubble, since the primary vortical action is outside, and causes a thinly stretched cylindrical, rather than toroidal shape.

The vortex in the wake of the drop tends to stretch the interface (and surface tension is not high enough to resist the stretching) which leads to an almost uniformly elongated backward bag. New eddies are formed due to flow separation at the edge to the "bag" and a toroidal rim is detached after some time from this bag. A drop too responds to Bond number, but the response is shown in terms of an
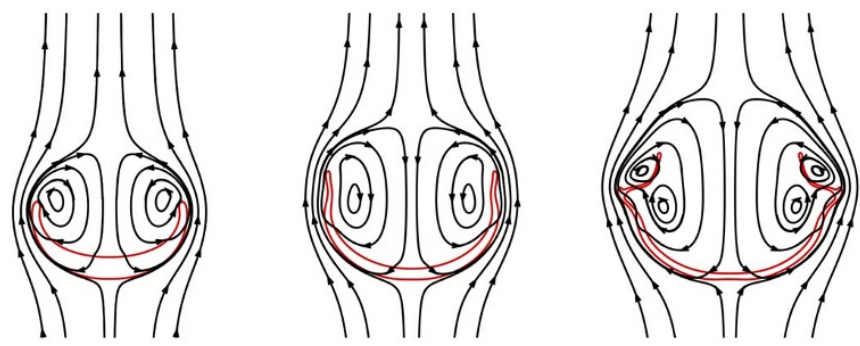

Figure $11 \mid$ Streamlines in and around the drop at time, $t=4.5,6$ and 7.5 respectively, for $G a=50, B o=5, r=10$ and $m=10$. 


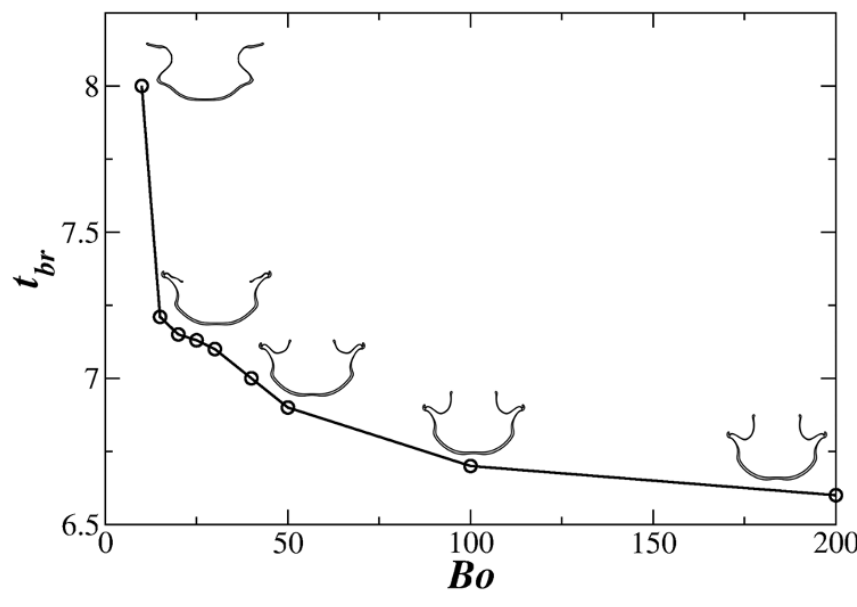

Figure $12 \mid$ Variation of break-up time with Bond number for $G a=50, r$ $=10$ and $m=10$.

early break-up at high Bond numbers, as seen in figure 12. The shape at break-up too evolves with the Bond number, as shown.

\section{Conclusions}

A bubble and a drop, starting from rest and moving under gravity in a surrounding fluid, cannot in general be designed to behave as one another's mirror images (one rising where the other falls). We have shown that the underlying mechanism which differentiates the dynamics is that the vorticity tends to concentrate in the lighter fluid, and this affects the entire dynamics, causing in general a thicker bubble and a thinner drop. However, if inertia is small, surface tension is large, and a drop is only slightly heavier than its surrounding fluid, a suitably chosen bubble can display dynamics similar to it. In this limit, the Hadamard solution can be exploited to design a bubble with its density and viscosity suitably chosen to yield the same acceleration at any time as a given drop. We are left with an interesting result: while a solid 'bubble' can never display a flow history which is the same as a solid 'drop', a Hadamard bubble can. Also, although density differences are small, the Boussinesq approximation cannot lead us to the closest bubble for a given drop.

We find numerically that a similarity in bubble and drop dynamics and shape is displayed up to moderate values of surface tension and inertia, so long as the density ratio is close to unity. In axisymmetric flow, the vorticity concentrates near the base of the bubble, which results in a pinch-off at the centre whereas the cup-like shape displayed by a drop, and the subsequent distortions of this shape due to the vorticity in the surrounding fluid, encourage a pinch-off at the sides. Bubbles of $\mathrm{Ga}$ higher than a critical value for a given $B o$ will break up at an inertial time between 2 and 3. For $G a$ or Bo just below the critical value, oscillations in shape of the same time scale occur before the steady state is achieved.

1. Taylor, T. \& Acrivos, A. On the deformation and drag of a falling viscous drop at low Reynolds number. J. Fluid Mech. 18, 466-476 (1964).

2. Hughes, R. \& Gilliland, E. The mechanics of drops. Chem. Eng. Prog. 48, 497-504 (1952)

3. Lane, W. \& Green, H. The mechanics of drops and bubbles. Surveys in Mechanics 162-215 (1956).

4. Wegener, P. P. \& Parlange, J.-Y. Spherical-cap bubbles. Annu. Rev. Fluid Mech. 5, 79-100 (1973).

5. De Gennes, P.-G., Brochard-Wyart, F. \& Quéré, D. Capillarity and wetting phenomena: drops, bubbles, pearls, waves (Springer, 2004).

6. Eggers, J. A brief history of drop formation. In Nonsmooth Mechanics and Analysis, 163-172 (Springer, 2006).

7. Legendre, D., Zenit, R. \& Velez-Cordero, J. R. On the deformation of gas bubbles in liquids. Phys. Fluids 24, 043303 (2012).

8. Tesař, V. Shape oscillation of microbubbles. Chem. Eng. J. 235, 368-378 (2014).
9. Chebel, N. A., Vejrazka, J., Masbernat, O. \& Risso, F. Shape oscillations of an oil drop rising in water: effect of surface contamination. J. Fluid Mech. 702, 533-542 (2012).

10. Lalanne, B., Tanguy, S. \& Risso, F. Effect of rising motion on the damped shape oscillations of drops and bubbles. Phys. Fluids 25 (2013)

11. Landel, J. R., Cossu, C. \& Caulfield, C. P. Spherical cap bubbles with a toroidal bubbly wake. Phys. Fluids 20, 122201 (2008).

12. Cano-Lozano, J., Bohorquez, P. \& Mart'inez-Bazán, C. Wake instability of a fixed axisymmetric bubble of realistic shape. Int. J. Multiphase Flow 51, 11-21 (2013).

13. Jalaal, M. \& Mehravaran, K. Fragmentation of falling liquid droplets in bag breakup mode. Int. J. Multiphase Flow 47, 115132 (2012).

14. Bhaga, D. \& Weber, M. E. Bubbles in viscous liquids: shapes, wakes and velocities J. Fluid Mech. 105, 61-85 (1981).

15. Davies, R. M. \& Taylor, G. I. The mechanics of large bubbles rising through extended liquids and through liquids in tubes. Proc. R. Soc. A. 200, 375-390 (1950).

16. Wu, M. \& Gharib, M. Experimental studies on the shape and path of small air bubbles rising in clean water. Phys. Fluids 14, L49 (2002).

17. Haberman, W. L. \& Morton, R. K. An experimental investigation of the drag and shape of air bubbles rising in various liquids. Tech. Rep., DTIC Document (1953).

18. Veldhuis, C., Arie, B. \& van Wijngaarden, L. Shape oscillations on bubbles rising in clean and in tap water. Phys. Fluids 20, 040705 (2008).

19. Abdel-Alim Ahmed, H. \& Hamielec, A. E. A theoretical and experimental investigation of the effect of internal circulation on the drag of spherical droplets falling at terminal velocity in liquid media. Ind. Eng. Chem. Fund. 14, 308-312 (1975).

20. Spells, K. A study of circulation patterns within liquid drops moving through a liquid. Proc. Phys. Soc. Lond. B 65, 541-546 (1952).

21. Krishna, P. M., Venkateswarlu, D. \& Narasimhamurty, G. S. R. Fall of liquid drops in water, terminal velocities. J. Chem. Eng. Data 4, 336340 (1959).

22. Hu, S. \& Kinter, R. C. The fall of single liquid drops through water. AIChE J. 1, 42-48 (1955).

23. Thorsen, G., Stordalen, R. M. \& Terjesen, S. G. On the terminal velocity of circulating and oscillating drops. Chem. Eng. Sci. 23, 413-426 (1968).

24. Wang, P. K. \& Pruppacher, H. R. Acceleration to terminal velocity of cloud and raindrops. J. Appl. Meteorol. 183, 275-280 (1977).

25. Hadamard, J. Mouvement permanent lent dune sphere liquide et visqueuse dans un liquide visqueux. CR Acad. Sci 152, 1735-1738 (1911).

26. Griffith, R. M. The effect of surfactants on the terminal velocity of drops and bubbles. Chem. Eng. Sci. 17, 10571070 (1962).

27. Klee, A. J. \& Treybal, R. E. Rate of rise or fall of liquid drops. AIChE J. 2, 444447 (1956).

28. Clift, R., Grace, J. \& Weber, M. Bubbles, drops and particles. 346 (Academic press, 1978).

29. Luo, H. \& Svendsen, H. F. Theoretical model for drop and bubble breakup in turbulent dispersions. AIChE J. 42, 1225-1233 (1996).

30. Liao, Y. \& Lucas, D. A literature review of theoretical models for drop and bubble breakup in turbulent dispersions. Chem. Eng. Sc. 64, 3389-3406 (2009).

31. Allen, H. S. The motion of a sphere in a viscous fluid. Lond. Edinb. Dubl. Phil. Mag. 50, 323-338 (1900).

32. Rybzynski, W. Über die fortschreitende bewegung einer flssigen kugel in einem zhen medium. Bull. Acad. Sci. (1911).

33. Hill, M. J. M. On a spherical vortex. Proc. R. Soc. Lond. A 185, 219-224 (1894)

34. Sussman, M. \& Smereka, P. Axisymmetric free boundary problems. J. Fluid Mech 341, 269-294 (1997).

35. Han, J. \& Tryggvason, G. Secondary breakup of axisymmetric liquid drops. i. acceleration by a constant body force. Phys. Fluids 11, 3650 (1999).

36. Brackbill, J., Kothe, D. B. \& Zemach, C. A continuum method for modeling surface tension. J. Comput. Phys 100, 335-354 (1992).

37. Ding, H., Spelt, P. D. M. \& Shu, C. Diffuse interface model for incompressible twophase flows with large density ratios. J. Comput. Phys 226, 2078-2095 (2007).

38. Uno, S. \& Kintner, R. Effect of wall proximity on the rate of rise of single air bubbles in a quiescent liquid. AIChE J. 2, 420-425 (1956).

39. Popinet, S. Gerris: a tree-based adaptive solver for the incompressible euler equations in complex geometries. J. Comput. Phys. 190, 572-600 (2003).

40. Mishra, M., De Wit, A. \& Sahu, K. C. Double diffusive effects on pressure-driven miscible displacement flows in a channel. J. Fluid Mech. 712, 579-597 (2012).

41. Kolár, V. 2d velocity-field analysis using triple decomposition of motion. In Proceedings of the 15th Australasian Fluid Mechanics Conference, CD-ROM, Paper AFMC00017 (http://www. aeromech. usyd. edu. au/15afmc), University of Sydney, Sydney, Australia, (2004).

42. Dixit, H. N. \& Govindarajan, R. Vortex-induced instabilities and accelerated collapse due to inertial effects of density stratification. J. Fluid Mech. 646, 415 (2010).

43. Widnall, S. E., Bliss, D. B. \& Tsai, C.-Y. The instability of short waves on a vortex ring. J. Fluid Mech. 66, 35-47 (1974).

44. Elzinga, E. R. \& Banchero, J. T. Some observations on the mechanics of drops in liquid-liquid systems. AIChE J. 7, 394-399 (1961).

45. Blanchard, D. C. Comments on the breakup of raindrops. J. Atmos. Sci. 19 119-120 (1962)

46. Hsiang, L. P. \& Faeth, G. M. Breakup criteria for fluid particles. Int. J. Multiphase Flow 18, 635-652 (1992). 
47. Kitscha, J. \& Kocamustafaogullari, G. Breakup criteria for fluid particles. Int. J. Multiphase Flow 15, 573-588 (1989).

48. Cohen, R. D. Effect of viscosity on drop breakup. Int. J. Multiphase Flow 20, 211-216 (1994).

49. Hinze, J. Fundamentals of the hydrodynamic mechanism of splitting in dispersion processes. AIChE J. 1, 289-295 (1955).

50. Ha, J.-W. \& Yang, S.-M. Breakup of a multiple emulsion drop in a uniform electric field. J. Colloid Interf. Sci. 213, 92-100 (1999).

51. Lee, C. S. \& Reitz, R. D. Effect of liquid properties on the breakup mechanism of high-speed liquid drops. Atomization Spray 11 (2001).

52. Villermaux, E. \& Bossa, B. Single-drop fragmentation determines size distribution of raindrops. Nature Phys. 5, 697-702 (2009).

53. Boulton-Stone, J. M. A note on the axisymmetric interaction of pairs of rising, deforming gas bubbles. Int. J. Multiphase Flow 21, 12371241 (1995).

54. Falcucci, G., Ubertini, S. \& Succi, S. Lattice Boltzmann simulations of phaseseparating flows at large density ratios: the case of doubly-attractive pseudopotentials. Soft Matter 6, 43574365 (2010).

55. Sbragaglia, M., Benzi, R., Biferale, L., Succi, S., Sugiyama, K. \& Toschi, F. Generalized lattice Boltzmann method with multirange pseudopotential. Phys. Rev. E 75, 026702 (2007).

56. Tomar, G., Fuster, D., Zaleski, S. \& Popinet, S. Multiscale simulations of primary atomization. Comput. Fluids 39, 1864-1874 (2010).

\section{Acknowledgments}

The TIFR Centre for Interdisciplinary Sciences and the Indian Institute of Technology, Hyderabad are thanked for support. K.S. also thanks the Department of Science and Technology, India for their financial support (Grant No: SR/FTP/ETA-85/2010).

\section{Author contributions}

M.T., K.S. and R.G. contributed to the theoretical and numerical analysis. All authors wrote and reviewed the manuscript.

\section{Additional information}

Supplementary information accompanies this paper at http://www.nature.com/ scientificreports

Competing financial interests: The authors declare no competing financial interests.

How to cite this article: Tripathi, M.K., Sahu, K.C. \& Govindarajan, R. Why a falling drop does not in general behave like a rising bubble. Sci. Rep. 4, 4771; DOI:10.1038/srep04771 (2014).

(c) (1) $(-)$ This work is licensed under a Creative Commons Attribution-NonCommercialNoDerivs 3.0 Unported License. The images in this article are included in the article's Creative Commons license, unless indicated otherwise in the image credit; if the image is not included under the Creative Commons license, users will need to obtain permission from the license holder in order to reproduce the image. To view a copy of this license, visit http://creativecommons.org/licenses/by-nc-nd/3.0/ 